\title{
Una breve síntesis de la evolución climática de la península ibérica durante los últimos 2000 años
}

\author{
DOI: https://doi.org/10.31978/639-18-002-5.02
}

\section{Santiago Giralt ${ }^{1}$, Ana Moreno ${ }^{2}$, Isabel Cacho ${ }^{3}$, Blas Valero-Garcés ${ }^{2}$}

\author{
${ }^{1}$ Instituto de Ciencias de la Tierra Jaume Almera (ICTJA-CSIC), Barcelona \\ ${ }^{2}$ Instituto Pirenaico de Ecología (IPE-CSIC), Zaragoza \\ ${ }^{3}$ GRC Geociències Marines, Dept. de Dinàmica de la Terra i de l'Oceà, Universitat de Barcelona, Barcelona
}

\begin{abstract}
Introducción
El único modo de conseguir una comprensión detallada de la variabilidad climática del pasado, y de la evolución a largo plazo de los modos climáticos a escala plurianualpluridecadal es a través de las reconstrucciones precisas de precipitación y de temperatura en el pasado. Estas reconstrucciones se pueden obtener a partir de un gran número de indicadores (en inglés, proxies) que se pueden medir en diferentes archivos naturales, tales como anillos de los árboles, secuencias sedimentarias marinas y lacustres, y registros de turberas y espeleotemas de cuevas, entre otros. Estas reconstrucciones permiten caracterizar las principales condiciones climáticas predominantes a diferentes escalas temporales y espaciales e identificar el comportamiento (estacionario o no estacionario) entre las diferentes variables climáticas.
\end{abstract}

La península ibérica está situada entre dos regiones climáticas: la Eurosiberiana (en el norte y noroeste) que se caracteriza por un clima frío y húmedo sin un marcado estiaje, y la Mediterránea, con una sequía estival muy marcada e inviernos relativamente frescos y húmedos. La geografía compleja que presenta la península ibérica, con un altiplano central segmentado y bordeado por una serie de cadenas montañosas que exceden los $2000 \mathrm{~m}$ de altitud, y su relativa extensa área costera, se traduce en la presencia de gradientes norte-sur y oeste-este decrecientes en precipitación y crecientes en temperatura. Debido a estas interacciones, existen en la península ibérica multitud de regímenes microclimáticos con una variada sensibilidad temporal y espacial a los principales modos climáticos que controlan la variabilidad climática de este territorio.

Es fundamental conocer la evolución plurianual-decadal de los principales modos climáticos del hemisferio norte, tales como la Oscilación del Atlántico Norte (NAO), el Atlántico Oriental (EA) y la Escandinava (SCAND) ya que controlan el clima de la península ibérica. Sin embargo, la falta de un conocimiento de su evolución a largo plazo limita nuestra capacidad de predecir su evolución futura, más aún en el actual contexto de calentamiento global. Para poder adquirir dicho conocimiento necesitamos datos robustos y fiables, ya sea procedentes de mediciones instrumentales o de modelos globales climáticos (GCM, en inglés), pero hay que tener en cuenta que solo las reconstrucciones procedentes de los archivos naturales del clima pasado nos dan esa perspectiva temporal a largo plazo. En este artículo presentamos un breve resumen de la evolución climática de la península ibérica de los últimos 2000 años obtenido a partir de la integración de diferentes conjuntos de datos de reconstrucciones climáticas mediante archivos marinos y continentales. La Figura 1 muestra la localización de las principales reconstrucciones paleoclimáticas disponibles en la península ibérica para este periodo de tiempo y las destacadas en la Figura 2 corresponden a un ejemplo del tipo de reconstrucciones paleoclimáticas disponibles en la actualidad. Las fluctuaciones climáticas de estos dos últimos milenios muestran la variabilidad reciente del Holoceno antes del Calentamiento Global (CG) y que incluye principalmente cuatro periodos: el Periodo Romano (PR, ca. 250 AC-500 AD), la Alta Edad Media (AEM, 500-900 AD), la Anomalía Climática Medieval (ACM, 900-1300 AD) y la Pequeña Edad de Hielo (PEH, 1300-1850 AD). La comparación de periodos cálidos en el pasado como el PR y ACM con el CG actual permitiría evaluar con más precisión los impactos relativos de las actividades antrópicas y de la variabilidad climática natural en el cambio climático actual y la evolución de los principales modos climáticos para finales del siglo XXI. La información relevante del clima en el pasado solo se obtiene a partir de registros bien datados con una elevada resolución temporal y una aproximación multiparamétrica robusta. En el caso de la península ibérica, además, estos registros tienen que cubrir transectos latitudinales y longitudinales para, así, capturar en su totalidad la historia de las múltiples regiones microclimáticas de la península ibérica. En la actualidad existen un gran número de reconstrucciones tanto marinas como continentales y se han llevado a cabo varios esfuerzos para sintetizar la evolución climática y sus principales tendencias en la península ibérica durante los últimos 2000 años, a varias escalas temporales y en diferentes ámbitos geográficos (Morellón et al., 2012; Moreno et al., 2012; Cisneros et al., 2016; Sánchez-López et al., 2016; Abrantes et al., 2017). A pesar de ello, aún existen lagunas geográficas significativas, tales como la zona central de la península ibérica o las zonas de alta montaña, lo que limita la obtención de una imagen más precisa de la evolución climática de la península ibérica para estos dos últimos milenios.

\section{El Periodo Romano (ca. 250 AC-500 AD)}

Las reconstrucciones climáticas marinas y continentales disponibles sugieren que, durante este periodo, las condiciones climáticas eran relativamente cálidas. Los registros 


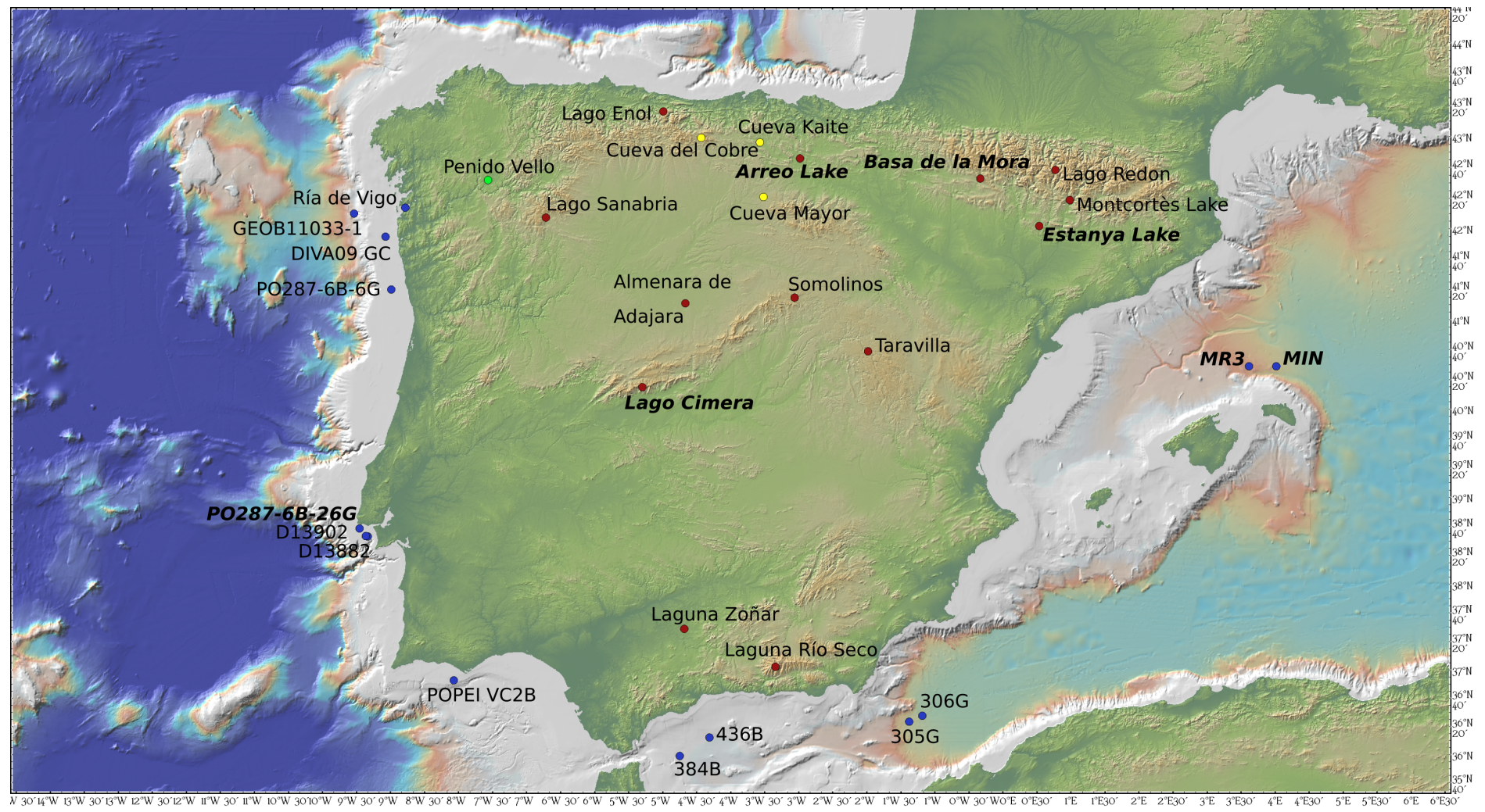

Figura 1. Mapa de la península ibérica con la localización de los principales registros actualmente disponibles para los últimos 2000 años. Los puntos azules marcan la posición de los registros marinos, los rojos representan las secuencias lacustres, los amarillos coresponden a los registros de espeleotemas en cuevas y los puntos verdes marcan las turberas. Los nombres en negrita y cursiva corresponden a los registros usados en la figura 2.

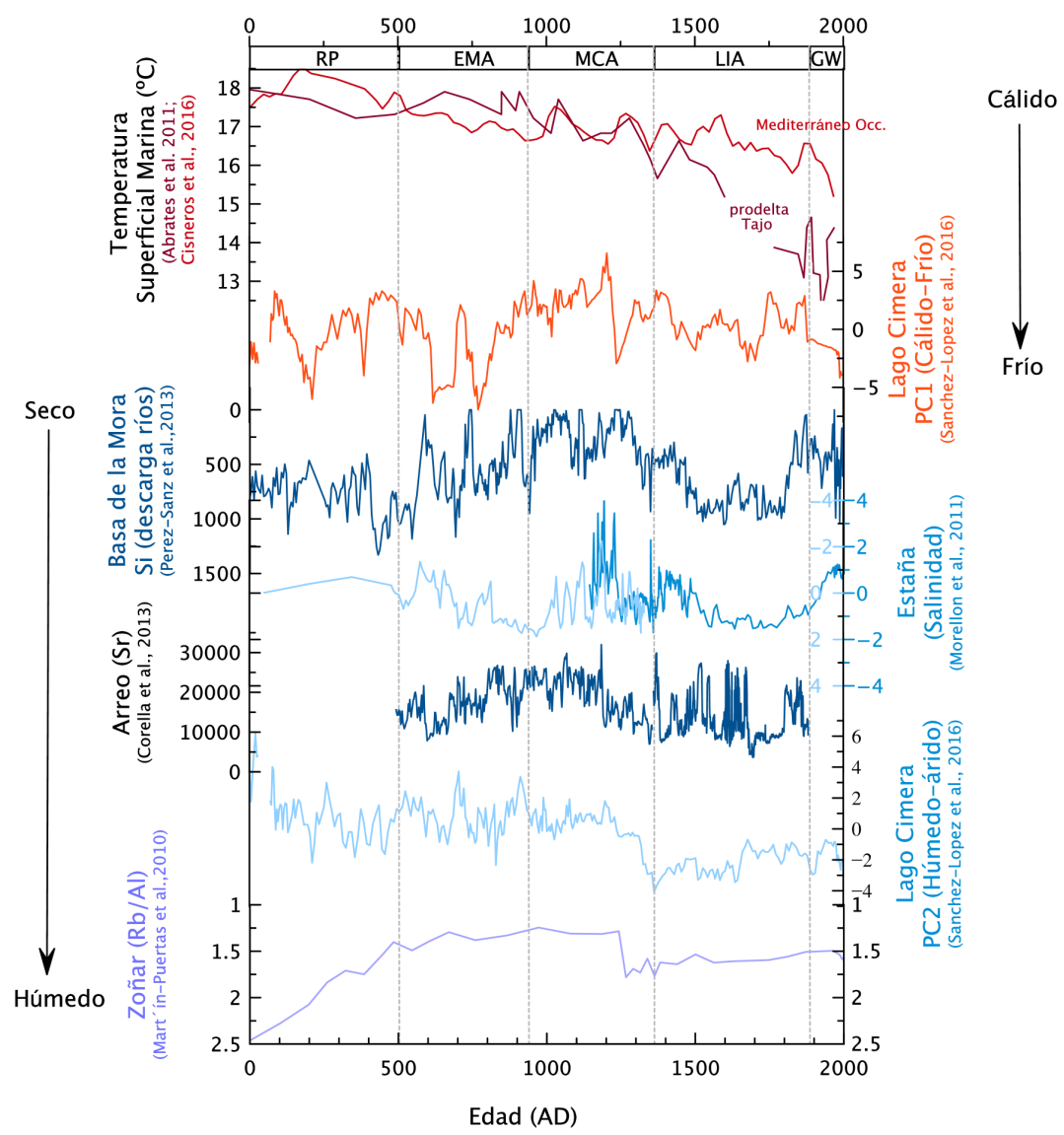

Figura 2. Selección de algunos de los registros que muestran los cambios de temperatura y humedad en la Península. La fuente de los datos se indica en la figura. 
de la temperatura superficial del mar de ambos lados de la península ibérica, tanto en el margen atlántico como en el sector baleárico, muestran que este periodo fue el más cálido de los últimos 2000 años (Abrantes et al., 2011; Cisneros et al., 2016). En el ámbito continental, se documenta un calentamiento a largo plazo en los Pirineos (Pla and Catalán, 2005) mientras que en la zona central de la península ibérica este periodo se caracterizó por una alternancia a escala de centenares de años y de décadas de fases frías y cálidas (Sánchez-López et al., 2016). En cuanto a la humedad, los registros disponibles muestran una distribución espacial relativamente compleja: mientras en el norte de la península ibérica dominaban condiciones áridas, la zona central de la península presentaba una alternancia de fases húmedas y áridas y en el sur predominaban condiciones más húmedas (Martín-Puertas et al., 2008; Morellón et al., 2012; Sánchez-López et al., 2016). Además, la comparación entre zonas de alta y baja montaña también pone de manifiesto un patrón hidrológico complejo. Así, mientras los registros situados en las localidades de alta montaña en los Pirineos orientales reflejan condiciones húmedas, los localizados en zonas de baja montaña más occidentales indican que prevalecieron condiciones de aridez (Morellón et al., 2011). Estos gradientes latitudinales y altitudinales podrían estar relacionados con la variabilidad regional pluviométrica, aunque la escasez de registros disponibles no permite realizar una evaluación de los posibles procesos climáticos responsables.

\section{La Alta Edad Media (500-900 AD)}

Este periodo también se caracteriza por un patrón espacial climático complejo. El noroeste de la península ibérica estuvo dominado por condiciones climáticas frías y húmedas (Jambrina-Enríquez et al., 2014) mientras que la zona oriental presentó condiciones más áridas de modo dominante, aunque con una heterogeneidad hidrológica mucho mayor. El balance entre evaporación y precipitación (E-P) disminuyó en el área mediterránea de Menorca (Cisneros et al., 2016) de forma consistente con la tendencia a la aridez presente en los Pirineos centrales (Morellón et al., 2011; Pérez-Sanz et al., 2013), aunque se ha identificado una gran variabilidad en las zonas centrales y meridionales de la península ibérica (Nieto-Moreno et al., 2011; Sánchez-López et al., 2016). De todas formas, algunas localidades situadas en los Pirineos sugerirían una mayor precipitación durante la AEM que en el periodo previo (Corella et al., 2013). Por tanto, en términos de humedad, el gradiente atlánticomediterráneo se mantendría aunque la falta de reconstrucciones climáticas robustas distribuidas a lo largo de la geografía peninsular impediría una caracterización más detallada de estos gradientes. Por otra parte, las condiciones térmicas de este periodo para toda la península ibérica serían más homogéneas, con un predominio de las condiciones climáticas más frías que durante el periodo anterior. Por ejemplo, en la zona mediterránea de Menorca se ha reconstruido un descenso térmico de entre 1 y $2{ }^{\circ} \mathrm{C}$ (Cisneros et al., 2016).

\section{La Anomalía Climática Medieval (900-1300 AD)}

La mayor disponibilidad de registros climáticos que cubren este periodo así como su mejor distribución espacial ha permitido una reconstrucción más precisa de sus condiciones climáticas (Moreno et al., 2012). Los registros marinos situados en el noroeste peninsular sugieren que la primera mitad de la ACM estuvo dominada por inviernos cálidos y primaveras y otoños frescos con grandes tormentas mientras que la segunda mitad de este periodo estuvo marcada por primaveras y otoños más cálidos y áridos (Abrantes et al., 2017). Por otro lado, en la zona mediterránea de Menorca (Cisneros et al., 2016) y del mar de Alborán (Nieto-Moreno et al., 2011) imperaron condiciones más áridas. En los registros continentales, se han documentado condiciones áridas en el norte (Martín-Chivelet et al., 2011; Morellón et al., 2012), centro (Sánchez-López et al., 2016) y sur de la península ibérica (Martín-Puertas et al., 2008). Algunas reconstrucciones climáticas llevadas a cabo en los Pirineos muestran condiciones más húmedas (Pla-Rabes and Catalán, 2011) o con un incremento en la frecuencia de tormentas (Corella et al., 2014), posiblemente relacionadas con factores locales, tales como la situación geográfica. Así, en cuanto a condiciones térmicas, casi todas las reconstrucciones climáticas realizadas a partir de registros continentales sugieren que toda la península ibérica estuvo dominada por condiciones cálidas.

\section{La Pequeña Edad de Hielo (1300-1850 AD)}

La LIA representa la última fase fría que tuvo lugar antes del calentamiento reciente de origen antropogénico. Las reconstrucciones climáticas marinas y continentales muestran condiciones más frías y húmedas pero con una estructura doble. Los registros del área de Menorca sugieren que la primera fase térmica se caracterizó por temperaturas superficiales marinas promedio más cálidas aunque con grandes oscilaciones, mientras que la segunda fase estuvo dominada por temperaturas más frescas y con oscilaciones de más alta frecuencia (Cisneros et al., 2016). Los registros marinos situados en la zona septentrional y central del océano Atlántico también muestran esta transición que se produce a lo largo de la PEH de condiciones cálidas a más frescas, pero con una tendencia sobreimpuesta de un enfriamiento intenso de cerca de unos $3{ }^{\circ} \mathrm{C}$ (Abrantes et al., 2017). Las secuencias del mar de Alborán indican un dominio de las condiciones húmedas con un patrón de oscilación a escala de décadas (Martín-Puertas et al., 2010; Nieto-Moreno et al., 2011). Por otra parte, los registros continentales del noroeste peninsular sugieren que la PEH se caracterizó por condiciones climáticas más húmedas y frescas con respecto a los periodos anteriores. El inicio de este periodo así como la intensidad de los cambios evidencian que existen claras diferencias regionales (Morellón et al., 2012; Corella et al., 2013; Pérez-Sanz et al., 2013; Oliva et al., 2018). Algunas reconstrucciones sugieren niveles de la lámina de agua de los lagos más elevados, especialmente durante la mitad y final del siglo XIX (Morellón et al., 2011). El inicio de la PEH en el registro del lago Montcortès se caracteriza por un incremento significativo de las lluvias de finales de finales de primavera-verano entre 1372 y $1452 \mathrm{AD}$, lo que sugeriría una frecuencia anómala de llegadas de frentes fríos de origen septentrional hacia latitudes más meridionales durante la estación cálida (Corella et al., 2016). Teniendo en cuenta la diferente resolución temporal de los modelos de edad de los registros, la coherencia entre los periodos de nivel del lago alto y la baja actividad solar indicaría que el control paleohidrológico de los lagos a escala de centenares de años se debería a causas climáticas. Las condiciones climáticas reconstruidas 
durante la PEH en el sistema Central (Sánchez-López et al., 2016) y en la cordillera Ibérica (Barreiro-Lostres et al., 2015) muestran que estarían dominadas por condiciones frías y húmedas con un incremento de eventos extremos (Moreno et al., 2008). El hecho de que estas oscilaciones decadales no aparezcan en todos los registros y cuando lo hacen la intensidad es variable refleja la diferente resolución temporal de los registros y/o sensibilidad del indicador empleado en la reconstrucción paleoclimática.

\section{Calentamiento Global (1850 AD-actualidad)}

Los registros meteorológicos instrumentales muestran de una forma clara que los últimos 150 años se han caracterizado por un progresivo incremento en la temperatura y una disminución en la precipitación como consecuencia de las actividades antropogénicas (Bladé et al., 2010). El incremento progresivo en la aridez se puede observar en la mayor parte de los registros continentales de la península ibérica, mientras que los registros marinos, especialmente en aquellos situados en el sureste peninsular, muestran un incremento en humedad (Nieto-Moreno et al., 2011). Con respecto a la temperatura, los registros continentales situados a baja altitud muestran una concordancia con los registros meteorológicos instrumentales, en los que se observa frecuentemente una tendencia hacia condiciones cada vez más cálidas y áridas. En cambio, algunos lagos de alta montaña (Sánchez-López et al., 2016) y varios registros marinos (Cisneros et al., 2016) registran un marcado descenso térmico, aunque los registros marinos situados en el golfo de León sí que capturan el calentamiento global (Sicre et al., 2016). La falta de registros con reconstrucciones robustas así como la aparición de diferentes interferencias de las actividades humanas con la evolución de los indicadores estudiados durante este periodo provoca que aún no exista una comparación robusta entre datos de indicadores y mediciones instrumentales. Existen varias iniciativas al respecto que permitirán obtener dichas comparaciones robustas en un futuro próximo.

\section{Mecanismos de forzamiento climático}

La variabilidad climática de la península ibérica reconstruida para los últimos 2000 años se ha explicado básicamente a partir de fluctuaciones en los modos climáticos y en la actividad solar y volcánica. La variabilidad de los principales modos climáticos, como la NAO, así como las variaciones en la insolación estival e invernal, se citan frecuentemente como los principales forzamientos para explicar tanto las tendencias climáticas a escala de miles y centenares de años como los cambios climáticos abruptos. Sin embargo, Comas-Bru y McDermott (2014) señalaron que, aparte del rol de un determinado modo climático, las interacciones entre ellos y la evolución espacio-temporal de dichas interacciones, son de vital importancia para explicar la variabilidad climática existente. El acople y desacople de la NAO con otros modos climáticos, como la EA y la SCAND, pueden traer como consecuencia cambios en los patrones espaciales de la temperatura y de la precipitación de invierno (Comas-Bru and McDermott, 2014). Además, estas interacciones también pueden tener como efecto que las distribuciones espaciales de los indicadores climáticos sean homogéneas o heterogéneas debido a la interacción de la NAO y la EA con el mismo u opuesto signo (Comas-Bru and McDermott, 2014; Bastos et al., 2016).
Estas interacciones climáticas también afectan a la península ibérica. Hernández et al. (2015) pusieron de manifiesto que la precipitación invernal de la península está básicamente controlada por la NAO, mientras que la EA es la principal responsable de la variabilidad térmica estival e invernal. El dominio de la fase positiva (negativa) de la NAO comporta el descenso (aumento) en la precipitación de invierno, mientras que la fase positiva (negativa) de la EA puede causar un aumento (disminución) en las temperaturas de invierno y verano. Así, los periodos con cambios en temperatura y precipitación con tendencias opuestas podrían estar causados por la interacción de estos dos modos climáticos durante periodos de mismo signo u opuesto.

Sánchez-López et al. (2016) propusieron que el signo de estos modos climáticos así como su interacción podría explicar la variabilidad espacio-temporal observada en la península ibérica para los últimos 2000 años. Los gradientes de humedad presentes durante los periodos PR y AEM serían, por tanto, la consecuencia de la interacción predominante de los modos climáticos de la NAO y EA durante las fases de signo opuesto $\left(\mathrm{NAO}^{+}-\mathrm{EA}^{-}\right.$y $\left.\mathrm{NAO}^{-}-\mathrm{EA}^{+}\right)$mientras que la distribución homogénea de la precipitación que sucedió durante los periodos de la ACM y la PEH se debería a la interacción de ambos modos climáticos durante la fase de mismo signo $\left(\mathrm{NAO}^{+}-\mathrm{EA}^{+}\right.$y $\left.\mathrm{NAO}^{-}-\mathrm{EA}^{-}\right)$. Por tanto, las principales condiciones climáticas durante el $\mathrm{PR}$ se corresponderían con un dominio de la interacción $\mathrm{NAO}^{-}-\mathrm{EA}^{+}$que se tradujo en inviernos cálidos y húmedos y veranos cálidos mientras que la climatología de la AEM estuvo controlada por el predominio de la interacción $\mathrm{NAO}^{+}-\mathrm{EA}^{-}$, con inviernos fríos y secos y veranos fríos. Por otra parte, el periodo de la ACM se asociaría a la interacción $\mathrm{NAO}^{+}-\mathrm{EA}^{+}$, con inviernos cálidos y secos y veranos cálidos mientras las condiciones climáticas de la PEH se debieron a la interacción $\mathrm{NAO}^{-}-\mathrm{EA}^{-}$, con inviernos húmedos y fríos y veranos fríos. Los registros marinos situados mar adentro de la costa portuguesa sugieren que las condiciones climáticas de la ACM también vendrían marcadas por cambios en la irradiancia solar total y que, por tanto, el modo climático SCAND podría ser el responsable del descenso significativo en la precipitación del norte de Portugal y de las bajas temperaturas de la superficie del mar en el sur (Abrantes et al., 2017).

\section{Conclusiones}

Las reconstrucciones climáticas continentales y marinas disponibles en la península ibérica para los últimos 2000 años muestran una evolución espacio-temporal de la precipitación y la temperatura compleja. Estas reconstrucciones ponen de manifiesto la presencia de microclimas, replicando hasta cierto punto la variabilidad climática actual peninsular, con una geografía compleja que condiciona una variabilidad espacial significativa. En general, la evolución climática de la península ibérica durante estos 2000 últimos años se puede subdividir en cuatro periodos climáticos: el Periodo Romano, la Alta Edad Media, la Anomalía Climática Medieval y la Pequeña Edad de Hielo, los cuales se caracterizan por patrones de precipitación y temperatura específicos. Las condiciones climáticas dominantes serían el resultado de la interacción de los principales modos climáticos (NAO, EA y SCAND), así como de fluctuaciones a largo y corto plazo de la insolación total de invierno y 
verano. Es necesario un mayor esfuerzo para poder obtener reconstrucciones climáticas multiparamétricas más robustas en zonas donde la cobertura geográfica es baja, como en la zona central peninsular y áreas de la costa septentrional de la Península. Además, es fundamental mejorar el conocimiento en la evolución a nivel decadal y de centenares de años de los principales modos climáticos, con especial énfasis en la EA y el SCAND, así como la evolución de la interacción entre ellos y con la irradiancia solar total.

\section{Bibliografía}

Abrantes, F., Rodrigues, T., Rufino, M., Salgueiro, E., Oliveira, D., Gomes, S., Oliveira, P., Costa, A., Mil-Homens, M., Drago, T., Naughton, F., 2017: The climate of the Common Era off the Iberian Peninsula. Clim. Past, 13, 1901-1918. doi:10.5194/cp-13-1901-2017.

Barreiro-Lostres, F., Brown, E., Moreno, A., Morellón, M., Abbott, M., Hillman, A., Giralt, S., Valero-Garcés, B., 2015: Sediment delivery and lake dynamics in a Mediterranean mountain watershed: Human-climate interactions during the last millennium (El Tobar Lake record, Iberian Range, Spain). Science of The Total Environment, 533, 506-519. doi:10.1016/j.scitotenv.2015.06.123.

Bastos, A., Janssens, I. A., Gouveia, C. M., Trigo, R. M., Ciais, P., Chevallier, F., Peñuelas, J., Rödenbeck, C., Piao, S., Friedlingstein, P., Running, S. W., 2016: European land $\mathrm{CO}_{2}$ sink influenced by NAO and East-Atlantic Pattern coupling. Nature Comms, 7, ncomms10315. doi:10.1038/ ncomms10315.

Blade, I., Castro, Y., Altava-Ortiz, V., Ancell, R., Argüeso, D., Barrera-Escoda, A., Brunet, M., Calvo, N., Errasti, I., Esteban-Parra, M. J., Fernández, J., Fortuny, D., Frías, M. D., Gallego, M. C., Gallego, D., Gámiz-Fortis, S. R., García-Herrer, R., Guijarro, J. A., Gutiérrez, J. M., Herrera, S., Izaguirre, C., Hidalgo-Muñoz, J. M., López-Moreno, J. I., Martín, M. L., Pons, M. R., Rasilla, D., Ribera, P., Rodrigo, F. S., Rodríguez-Puebla, C., Vicente-Serrano, S. M., 2010: Atmospheric trends in the Iberian Peninsula during the instrumental period in the context of natural variability. In F. Perez and R. Boscolo (eds.): Climate in Spain: Past, present and future. Regional climate change assessment report. 25-42 pp.

Cisneros, M., Cacho, I., Frigola, J., Canals, M., Masqué, P., Martrat, B., Casado, M., Grimalt, J. O., Pena, L. D., Margaritelli, G., Lirer, F., 2016: Sea surface temperature variability in the central-western Mediterranean Sea during the last 2700 years: a multi-proxy and multi-record approach. Clim. Past, 12, 849-869. doi:10.5194/cp-12-849-2016.

Comas-Bru, L., McDermott, F., 2014: Impacts of the EA and SCA patterns on the European twentieth century NAOwinter climate relationship. Q. J. R. Meteorol. Soc., 140, 354-363. doi:10.1002/qj.2158.

Corella, J. P., Benito, G., Rodríguez-Lloveras, X., Brauer, A., Valero-Garcés, B. L., 2014: Annually-resolved lake record of extreme hydro-meteorological events since AD 1347 in NE Iberian Peninsula. Quaternary Science Reviews, 93, 77-90. doi:10.1016/j.quascirev.2014.03.020.
Corella, J. P., Stefanova, V., El Anjoumi, A., Rico, E., Giralt, S., Moreno, A., Plata-Montero, A., Valero-Garcés, B. L., 2013: A 2500-year multi-proxy reconstruction of climate change and human activities in northern Spain: The Lake Arreo record. Palaeogeography, Palaeoclimatology, Palaeoecology, 386, 555-568. doi:10.1016/j. palaeo.2013.06.022.

Corella, J. P., Valero-Garcés, B. L., Vicente-Serrano, S. M., Brauer, A., Benito, G., 2016: Three millennia of heavy rainfalls in Western Mediterranean: frequency, seasonality and atmospheric drivers. Scientific Reports, 6. doi:10.1038/ srep38206.

Hernández, A., Trigo, R. M., Pla-Rabes, S., Valero-Garcés, B. L., Jerez, S., Rico-Herrero, M., Vega, J. C., JambrinaEnríquez, M., Giralt, S., 2015: Sensitivity of two Iberian lakes to North Atlantic atmospheric circulation modes. Clim. Dyn., 45, 3403-3417. doi:10.1007/s00382-0152547-8.

Jambrina-Enríquez, M., Rico, M., Moreno, A., Leira, M., Bernárdez, P., Prego, R., Recio, C., Valero-Garcés, B. L., 2014: Timing of deglaciation and postglacial environmental dynamics in NW Iberia: the Sanabria Lake record. Quaternary Science Reviews 94, 136-158. doi:10.1016/j. quascirev.2014.04.018.

Martín-Chivelet, J., Muñoz-García, M. B., Edwards, R. L., Turrero, M. J., Ortega, A. I., 2011: Land surface temperature changes in Northern Iberia since $4000 \mathrm{yr} B P$, based on $\delta 13 \mathrm{C}$ of speleothems. Global Planet. Change. doi:10.1016/ j.gloplacha.2011.02.002.

Martín-Puertas, C., Jiménez-Espejo, F., Martínez-Ruiz, F., Nieto-Moreno, V., Rodrigo, M., Mata, M. P., ValeroGarcés, B.L., 2010: Late Holocene climate variability in the southwestern Mediterranean region: an integrated marine and terrestrial geochemical approach. Clim. Past, 6, 807-816. doi:10.5194/cp-6-807-2010.

Martín-Puertas, C., Valero-Garcés, B. L., Mata, P., González-Sampériz, P., Bao, R., Moreno, A., Stefanova, V., 2008: Arid and Humid Phases in Southern Spain during the last 4000 Years: The Zoñar Lake Record, Córdoba. The Holocene, 40, 195-215.

Morellón, M., Pérez-Sanz, A., Corella, J. P., Büntgen, U., Catalán, J., González-Sampériz, P., González-Trueba, J. J., López-Sáez, J. A., Moreno, A., Pla-Rabes, S., Saz-Sánchez, M. Á., Scussolini, P., Serrano, E., Steinhilber, F., Stefanova, V., Vegas-Vilarrúbia, T., Valero-Garcés, B., 2012: A multiproxy perspective on millennium-long climate variability in the Southern Pyrenees. Clim. Past, 8, 683-700. doi:10.5194/cp-8-683-2012.

Morellón, M., Valero-Garcés, B., González-Sampériz, P., Vegas-Vilarrúbia, T., Rubio, E., Rieradevall, M., DelgadoHuertas, A., Mata, P., Romero, Ó., Engstrom, D. R., López-Vicente, M., Navas, A., Soto, J., 2011: Climate changes and human activities recorded in the sediments of Lake Estanya (NE Spain) during the Medieval Warm Period and Little Ice Age. Journal of Paleolimnology, 46, 423-452. doi:10.1007/s10933-009-9346-3. 
Morellón, M., Valero-Garcés, B., Vegas-Vilarrúbia, T., González-Sampériz, P., Romero, Ó., Delgado-Huertas, A., Mata, P., Moreno, A., Rico, M., Corella, J. P., 2009: Lateglacial and Holocene palaeohydrology in the western Mediterranean region: The Lake Estanya record (NE Spain). Quaternary Science Reviews, 28, 2582-2599.

Moreno, A., Pérez, A., Frigola, J., Nieto-Moreno, V., Rodrigo-Gámiz, M., Martrat, B., González-Sampériz, P., Morellón, M., Martín-Puertas, C., Corella, J. P., Belmonte, Á., Sancho, C., Cacho, I., Herrera, G., Canals, M., Grimalt, J. O., Jiménez-Espejo, F., Martínez-Ruiz, F., VegasVilarrúbia, T., Valero-Garcés, B. L., 2012: The Medieval Climate Anomaly in the Iberian Peninsula reconstructed from marine and lake records. Quat. Sci. Rev., 43, 16-32. doi:10.1016/j.quascirev.2012.04.007.

Moreno, A., Valero-Garcés, B. L., González-Sampériz, P., Rico, M., 2008: Flood response to rainfall variability during the last 2000 years inferred from the Taravilla Lake record (Central Iberian Range, Spain). Journal of Paleolimnology, 40, 943-961.

Nieto-Moreno, V., Martínez-Ruiz, F., Giralt, S., JiménezEspejo, F., Gallego-Torres, D., Rodrigo-Gámiz, M., García-Orellana, J., Ortega-Huertas, M., de Lange, G. J., 2011: Tracking climate variability in the western Mediterranean during the Late Holocene: a multiproxy approach. Clim. Past Discuss., 7, 635-675. doi: 10.5194/ cpd-7-635-2011.

Oliva, M., Ruiz-Fernández, J., Barriendos, M., Benito, G., Cuadrat, J. M., Domínguez-Castro, F., García-Ruiz, J. M., Giralt, S., Gómez-Ortiz, A., Hernández, A., López-Costas, O., López-Moreno, J. I., López-Sáez, J. A., MartínezCortizas, A., Moreno, A., Prohom, M., Saz, M. A., Serrano, E., Tejedor, E., Trigo, R., Valero-Garcés, B., VicenteSerrano, S., 2018: The Little Ice Age in Iberian mountains. Earth Science Reviews, 177, 175-208. doi: 10.1016/ j.earscirev.2017.11.010.
Pérez-Sanz, A., González-Sampériz, P., Moreno, A., ValeroGarcés, B., Gil-Romera, G., Rieradevall, M., Tarrats, P., Lasheras-Álvarez, L., Morellón, M., Belmonte, A., Sancho, C., Sevilla-Callejo, M., Navas, A., 2013: Holocene climate variability, vegetation dynamics and fire regime in the central Pyrenees: the Basa de la Mora sequence (NE Spain). Quaternary Science Reviews, 73, 149-169. doi:10.1016/ j.quascirev.2013.05.010.

Pla, S., \& Catalán, J., 2005: Chrysophyte cysts from lake sediments reveal the submillennial winter/spring climate variability in the northwestern Mediterranean region throughout the Holocene. Clim. Dyn., 24 (2), 263. doi: 10.1007/s00382-004-0482-1.

Pla-Rabes, S., Catalán, J., 2011: Deciphering chrysophyte responses to climate seasonality. J. Paleolimnol., 46, 139. doi: 10.1007/s10933-011-9529-6.

Rodrigues, T., Grimalt, J. O., Abrantes, F. G., Flores, J. A., \& Lebreiro, S. M., 2009: Holocene interdependences of changes in sea surface temperature, productivity, and fluvial inputs in the Iberian continental shelf (Tagus mud patch). Geochemistry, Geophysics, Geosystems, 10 (7), doi: 10.1029/2008GC002367.

Sánchez-López, G., Hernández, A., Pla-Rabes, S., Trigo, R. M., Toro, M., Granados, I., Sáez, A., Masqué, P., Pueyo, J. J., Rubio-Inglés, M. J., Giralt, S., 2016: Climate reconstruction for the last two millennia in central Iberia: The role of East Atlantic (EA), North Atlantic Oscillation (NAO) and their interplay over the Iberian Peninsula. Quaternary Science Reviews, 149, 135-150. doi:10.1016/ j.quascirev.2016.07.021.

Sicre, M.-A., Jalali, B., Martrat, B., Schmidt, S., Bassetti, M.-A., Kallel, N., 2016: Sea surface temperature variability in the North Western Mediterranean Sea (Gulf of Lion) during the Common Era. Earth Planet. Sci. Lett., 456, 124-133. doi: 10.1016/j.epsl.2016.09.032. 